\title{
PATOGENICIDADE DE Verticillium lecanii AO PULGÃO-DO-PINUS ${ }^{1}$
}

\author{
Elisângela de Souza Loureiro², Nádia Cristina de Oliveira², Carlos Frederico Wilcken² e Antonio Batista Filho ${ }^{3}$
}

\begin{abstract}
RESUMO - Avaliou-se a patogenicidade do isolado IBCB 473 de Verticillium lecanii no pulgão-do-pinus Cinara atlantica (Hemiptera, Aphididae), inseto-praga em plantios de Pinus spp. O delineamento experimental utilizado foi o inteiramente casualizado, com cinco repetições por tratamento e cada repetição constituída de uma placa de Petri ( $9 \mathrm{~cm}$ de diâmetro) contendo 10 ninfas. Foram testadas suspensões de inóculo nas concentrações de $1,0^{\prime} 10^{6} ; 0,5^{\prime} 10^{7} ; 1,0^{\prime} 10^{7} ; 0,5^{\prime} 10^{8} ;$ e $1,0^{\prime} 10^{8}$ conídios $/ \mathrm{mL}$, pulverizando-se $1 \mathrm{~mL}$ sobre as ninfas. Como tratamento-testemunha, utilizou-se água esterilizada. As placas de Petri foram mantidas em câmara climatizada a $25 \pm 1{ }^{\circ} \mathrm{C}, 70 \pm 10 \%$ de UR e fotofase de 12 horas. As avaliações foram realizadas diariamente, anotando-se a mortalidade dos indivíduos de cada tratamento. A dose mais eficiente foi de $1,0 \times 10^{8}$ conídios/ $\mathrm{mL}$, causando mortalidade de $86,0 \%$ após cinco dias da pulverização. Os valores de $\mathrm{TL}_{50}$ das concentrações utilizadas foram de 5,$82 ; 5,24 ; 4,60 ; 4,34 ;$ e 3,83 dias, respectivamente.
\end{abstract}

Palavras-chave: Fungo entomopatogênico, controle biológico, praga florestal.

\section{PATHOGENICITY OF Verticillium lecanii TO PINE APHID}

\begin{abstract}
The objective of this work was to evaluate the pathogenicity of the entomopathogenic fungus Verticillium lecanii isolate IBCB 473 to the aphid Cinara atlantica (Hemiptera: Aphididae), insect pest in Pinus spp plantations. The trials were conduced in a completely randomized design, treatments with 5 repetitions, and each repetition consisting of one Petri dish (9 cm of diameter) containing 10 nymphs. Suspensions adjusted to concentrations of $1,0^{\prime} 10^{6} ; 0,5^{\prime} 10^{7} ; 1,0^{\prime} 10^{7} ; 0,5^{\prime} 10^{8}$ e $1,0^{\prime} 10^{8}$ conidia/mL were tested by spraying $1 \mathrm{~mL}$ over the nymphs. Sterilized water was used as control treatment. The Petri dishes were maintained at $25 \pm 1{ }^{\circ} \mathrm{C}, 70 \pm 10 \% \mathrm{RH}$ and 12-hour photophase. The evaluations were carried out daily, recording the individual mortality of each treatment. The best concentration was $1,0 \times 10^{8}$ conidia/mL with mortality of $86,0 \%$ after five days. $L T_{50}$ for each concentration was 5,82; 5,24; 4,60; 4,34 and 3,83 days, respectively.
\end{abstract}

Key Words: entomopathogenic fungi, biological control, forest pest.

\section{INTRODUÇÃO}

A área plantada com Pinus aumentou rapidamente nas últimas duas décadas na América do Sul. Atualmente, o Brasil dispõe de aproximadamente dois milhões de hectares plantados com Pinus (SBS, 1998).

Muitos pulgões são pragas conhecidas em áre- as florestais (DREES, 1993). As plantas atacadas, que podem ser mudas ou árvores de 20 anos, apresentam descoloração (clorose) e deformação das acículas, devido à picada de alimentação, ocorrendo à sua queda prematura e redução no desenvolvimento da planta (OTTATI, 1999; PENTEADO et al., 2000).

No Brasil, detectou-se pela primeira vez a ocorrência

\footnotetext{
${ }^{1}$ Recebido para publicação em 15.9.2003 e aceito para publicação em 10.8.2004.

${ }^{2}$ Departamento de Produção Vegetal da Faculdade de Ciências Agronômicas - UNESP, Campus de Botucatu, Cx. Postal 237, CEP 18603-970, Botucatu-SP.E-mail: <lis_loureiro@yahoo.com.br>

${ }^{3}$ Instituto Biológico - C.P. 70, CEP 13001-970, Campinas, SP.
} 
de afídeos do gênero Cinara, representado pelas espécies C. pinivora (WILSON, 1919) (Hemiptera: Aphididae), em 1996, infestando plantios de Pinus elliottii Engelm. e Pinus taeda Linn. nos municípios de Cambará do Sul (RS) e Lages (SC) (IEDE et al., 1998).

Os agentes de controle biológico vêm ganhando destaque no controle de insetos-praga da agricultura e da floresta. Nas últimas duas décadas, pesquisas foram publicadas sobre a relação entre agrotóxicos e casos de mutação, câncer e outras doenças em humanos, danos em animais selvagens e toxicidade às plantas (KHACHATOURIANS, 1986).

Determinadas características, como esporulação e germinação rápida, permitem que o processo de infecção seja completado em poucas horas, com grande produção de conídios, os quais maximizam a dispersão dos patógenos. Os fungos agentes microbianos são de extrema importância para o controle de algumas pragas que apresentam alta capacidade reprodutiva e ciclo de vida reduzido (MILNER, 1997).

Verticillium lecanii (Deuteromicotina: Hyphomycetes) (Zimm.) Viègas é um patógeno utilizado no controle de artrópodes. O espectro de espécies que ele ataca é amplo e inclui insetos homópteros (afídeos, coccídeos e aleirodídeos), entre outros grupos (STEENBERG e HUMBER, 1999). Seus produtos comerciais estão disponíveis na Europa: Mycotal (contra aleirodídeos e tripes) e Vertalec (contra afídeos). No Brasil este fungo ainda não foi formulado para comercialização. Mycotal é um pó molhável que foi utilizado no controle de Trialeurodes vaporariorum (Hemiptera: Aleyrodidae) e Frankliniella occidentalis (Thisanoptera: Thripidae), resultando, respectivamente, na redução de 90 e $60 \%$ da população dessas pragas, após aplicações semanais do produto em plantações de pepino e tomate na Holanda (Van der SCHAAF et al., 1990; ROVESTI et al., 1997).

Os danos causados por pulgões são consideravelmente reduzidos pelas epizootias naturais de doenças fúngicas, que podem ser consideradas como componentes eficientes do controle biológico natural, principalmente em altas densidades populacionais da praga e quando existem condições ambientais convenientes (MILNER, 1997). O fungo entomopatogênico $V$. lecanii foi registrado pela primeira vez atacando $C$. pinivora em $P$. elliottii em Lages, SC (PENTEADO et al., 2001) e C. atlantica (Hemiptera: Aphididae), atacando $P$. taeda em Nova Campina, SP (OLIVEIRA et al., 2002).

Assim, este trabalho teve por objetivo avaliar a patogenicidade do isolado IBCB 473 do fungo entomopatogênico V. lecanii ao pulgão-do-pinus $C$. atlantica, em condições de laboratório.

\section{MATERIAL E MÉTODOS}

O estudo foi realizado no Laboratório de Controle Biológico do Instituto Biológico, sediado em Campinas, SP. Foi utilizado o fungo entomopatogênico V. lecanii isolado IBCB 473 sobre ninfas de $C$. atlantica. As ninfas, provenientes de coletas realizadas em áreas reflorestadas com essa espécie arbórea, pertencentes à Orsa Papel e Celulose S/A, localizadas nos municípios de Buri e Nova Campina, SP, foram trazidas em caixas térmicas contendo ponteiros de $P$. taeda até o laboratório. Os pulgões foram colocados sobre um ponteiro de $P$. taeda medindo 8 cm de comprimento envolvido nas extremidades por um pedaço de algodão umedecido com água e acondicionados no interior de placas de Petri, mantendose, assim, as condições ideais para que o inseto e o fungo se desenvolvessem. O experimento foi conduzido com cinco repetições por tratamento, sendo cada repetição constituída de uma placa de Petri com 10 insetos, perfazendo um total de 50 insetos por tratamento, em delineamento experimental inteiramente casualizado.

Para a realização do bioensaio, o isolado IBCB 473 de V. lecanii (isolado de $C$. atlantica proveniente de Nova Campina, SP), foi multiplicado por meio de semeadura até a obtenção de culturas puras em placas cheias (conidiogênese), com alça de platina e posterior espalhamento com alça de Drigalsky, em placas de Petri ( $9 \mathrm{~cm}$ de diâmetro) contendo meio completo (MC), descrito por Alves et al. (1998).

Após a repicagem, as placas foram transferidas para incubação em câmara B.O.D. a $25 \pm 1{ }^{\circ} \mathrm{C}$ e fotofase de 12 horas, por um período de oito dias. Posteriormente, foram armazenadas em geladeira $\left(4^{\circ} \mathrm{C}\right)$ até a utilização nos experimentos. Após esse período, os conídios foram retirados por meio de raspagem com alça metálica e utilizados nas suspensões.

A contagem do número de conídios foi feita em 
câmara de Neubauer em microscópio ótico. As suspensões de conídios foram preparadas a partir da conidiogênese do fungo produzida em meio de cultura completo (MC) com água destilada estéril e espalhante adesivo (Tween 80 ) a $0,1 \%$.

A inoculação de V. lecanii foi feita através da aspersão de $1 \mathrm{~mL}$ de suspensão a concentração de $1,0^{\prime} 10^{6} ; 0,5^{\prime} 10^{7} ; 1,0^{\prime} 10^{7} ; 0,5^{\prime} 10^{8} ;$ e $1,0^{\prime} 10^{8}$ conídios/ $\mathrm{mL}$, sobre o ponteiro contendo os pulgões. A aplicação foi feita por uma torre de Potter adaptada, com pressão de 15 libras/pol ${ }^{2}$. Os ponteiros foram trocados a cada dois dias. No tratamento-testemunha foi adicionada água esterilizada $(1 \mathrm{~mL})$ nos ponteiros de P. taeda. As placas de Petri contendo os pulgões foram vedadas com filme de pvc perfurado e mantidas em câmara climatizada à temperatura de $25 \pm 1{ }^{\circ} \mathrm{C}, 70 \pm 10 \%$ de UR com fotofase de 12 horas. O filme de pvc foi trocado diariamente.

As avaliações foram realizadas diariamente, anotando-se a mortalidade dos indivíduos de cada tratamento. Os indivíduos mortos foram desinfestados com álcool 70\%, hipoclorito de sódio 30\% e água destilada. Em seguida, foram colocados em câmaras úmidas a $26 \pm 1{ }^{\circ} \mathrm{C}, 70 \pm 10 \%$ e fotofase de 12 horas, para confirmação da mortalidade causada pelo patógeno, através da observação do crescimento micelial e conidiogênese no cadáver.

Os dados obtidos foram submetidos à análise de
Probit para obtenção dos valores de $\mathrm{TL}_{50}$ (em dias) nos diversos tratamentos.

\section{RESULTADOS E DISCUSSÃO}

A atividade patogênica do fungo $V$. lecanii foi observada através dos tempos letais $\left(\mathrm{TL}_{50}\right)$. De maneira geral, os tempos letais foram decrescentes à medida que aumentou a concentração de inóculo (Tabela 1/ Table 1).

Ocorreu diferença significativa entre as concentrações testadas, observando-se os valores dos intervalos de confiança obtidos, exceto na dosagem de $0,5 \times 10^{8}$ conídios/mL. A dosagem de $1,0 \times 10^{8}$ conídios/mL de $V$. lecanii foi a mais eficiente, com $\mathrm{TL}_{50}$ de 3,83 dias, seguida da dosagem de $0,5 \times 10^{8}$ conídios/mL com $\mathrm{TL}_{50}$ de 4,34 dias. Esses dados estão de acordo com os observados por Loureiro (2001), que testou cinco concentrações desse fungo sobre as espécies de pulgões Aphis gossypii (Hemiptera: Aphididae) e Myzus persicae (Hemiptera: Aphididae), sendo a dosagem de $0,5 \times 10^{8}$ conídios $/ \mathrm{mL}$ a mais eficiente, com $\mathrm{TL}_{50}$ de 2,59 dias e a dosagem de 1,0 x $10^{8}$ conídios/mL com $\mathrm{TL}_{50}$ de 2,81 dias. Os dados obtidos por Hall (1982), em experimentos com pepino em casa de vegetação, para controlar A. gossypii, em que foi utilizada a formulação comercial Vertalec, desse fungo, também foram semelhantes aos encontrados no presente estudo.

Tabela 1 - Tempos letais medianos $\left(\mathrm{TL}_{50}\right)$ em dias, equações de regressão linear e valores de $x^{2}$ obtidos pela análise de Probit para Verticillium lecanii

Tables 1 -Mean lethal time ( $\left.L T_{50}\right)$ in days, regression linear equation and $x^{2}$ value obtained by analisys of Probit by Verticillium lecanii

\begin{tabular}{|c|c|c|c|c|}
\hline $\begin{array}{l}\text { Concentração } \\
\text { (conídios/mL) }\end{array}$ & $\mathrm{TL}_{50}$ & IC & Equação & $x^{2}$ \\
\hline $1,0 \times 10^{6}$ & 5,82 & $(5,64 ; 6,00)$ & $Y=2,15+3,72 \cdot \log x$ & 1,11 \\
\hline $0,5 \times 10^{7}$ & 5,24 & $(4,90 ; 5,62)$ & $Y=1,85+4,37 \cdot \log x$ & 4,41 \\
\hline $1,0 \times 10^{7}$ & 4,60 & $(4,25 ; 4,98)$ & $Y=2,13+4,34 \log x$ & 5,24 \\
\hline $0,5 \times 10^{8}$ & 4,34 & $(4,09 ; 4,61)$ & $Y=1,91+4,85 \cdot \log x$ & 2,67 \\
\hline $1,0 \times 10^{8}$ & 3,83 & $(2,34 ; 3,37)$ & $Y=1,86+5,37 \cdot \log x$ & 5,45 \\
\hline
\end{tabular}

$* x^{2}$ significativo $(\mathrm{P}<0,05)$.

* IC= intervalo de confiança. 
Foi observado um acréscimo nos valores de mortalidade acumulada confirmada em função da concentração de conídios e do tempo, sendo os maiores valores observados a partir do $5^{0}$ dia após a pulverização. A concentração de $1,0 \times 10^{8}$ conídios $/ \mathrm{mL}$ proporcionou às ninfas de $C$. atlantica mortalidade ao redor de $86 \%$ após cinco dias da infecção (Figura 1/Figure 1). Esses dados são concordantes com os de Fournier e Brodeur (1999), que investigaram as espécies de pulgões Macrosiphum euphorbiae (Hemiptera: Aphididae), M. persicae e Nasonovia ribisnigri (Hemiptera: Aphididae), que atacam alface em casa de vegetação, sendo $V$. lecanii altamente patogênico nas três espécies de pulgões estudadas em laboratório. Milner e Lutton (1986) obtiveram resultados em 96 horas após a inoculação de Vertalec em $M$. persicae, com uma mortalidade de 94,5\%, em experimentos de laboratório com umidade relativa ao redor de $100 \%$.

Dados também semelhantes aos do presente estudo (Figura 1/Figure 1) foram encontrados por Hall e Burges (1979), em cultivos de crisântemo em bioensaios de laboratório. Entretanto, Loureiro (2001) observou que após oito dias da infecção em ninfas de $A$. gossypii ocorreu mortalidade ao redor de $100 \%$ em todas as concentrações testadas. Van der Schaaf et al. (1990) observaram o controle de mosca-branca $T$. vaporariorum e tripes $F$. occidentalis com mortalidade de aproximadamente de 90 e $60 \%$, em cultivos de tomate e pepino, ocorrendo pequena diferença entre as concentrações testadas. Nas condições de laboratório, Vertalec controlou todas as espécies de afídeos testadas, e em condições ideais no laboratório pode chegar a $100 \%$ de infecção na população entre quatro e cinco dias (QUINLAN, 1988). Esse mesmo autor relatou ainda que, em comparação com outros fungos entomopatogênicos, V. lecanii é mais efetivo, mais barato de ser produzido, infecta tanto os adultos quanto as larvas de espécies de mosca branca e propaga-se bem no interior da população de pragas causando epizootia.

Foi escolhida a mortalidade confirmada como parâmetro para se estudar o comportamento da melhor concentração devido ao fato de que os fungos, como agentes de controle biológico, diferem fundamentalmente dos produtos fitossanitários de natureza química, pela capacidade de aumento da densidade do patógeno por meio da dispersão do inóculo secundário, repetindo o ciclo através da população hospedeira (HAJEK e St. LEGAR, 1994). A capacidade de colonização do patógeno, superando todos os agentes competidores presentes no inseto, e a capacidade de esporulação são fatores determinantes para disseminação do patógeno no ambiente (NEVES, 1998).

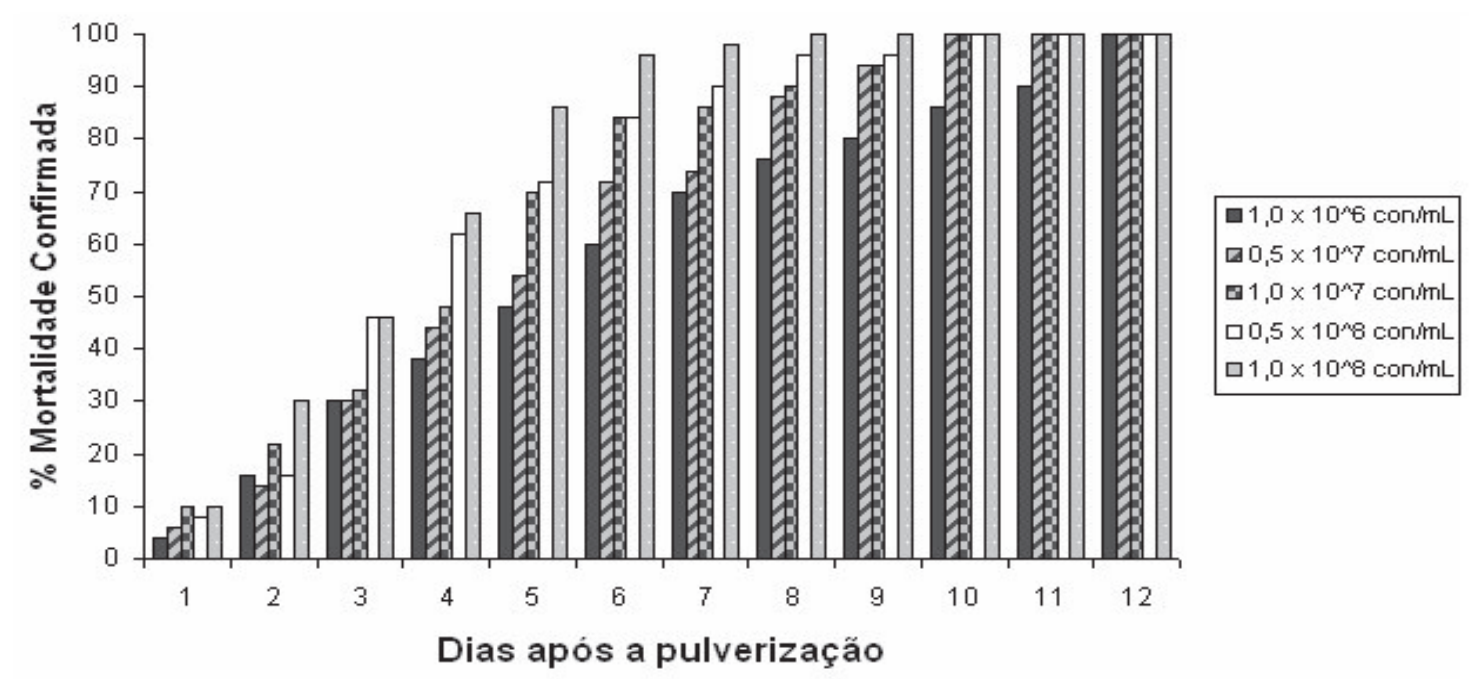

Figura 1 - Mortalidade acumulada e confirmada de Cinara atlantica após a inoculação com o fungo entomopatogênico Verticillium lecanii e incubação a $25 \pm 1{ }^{\circ} \mathrm{C}$ e $70 \pm 10 \%$; com fotofase de 12 horas.

Figure 1-Cumulative and confirmed mortality of Cinara atlantica after inoculation with entomopathogenic fungus Verticillium lecanii and incubation at $25 \pm 1{ }^{\circ} \mathrm{C}, 70 \pm 10 \% \mathrm{RH}$; 12-hour photophase.

R. Árvore, Viçosa-MG, v.28, n.5, p.765-770, 2004

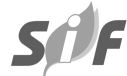




\section{CONCLUSÕES}

A espécie de pulgão $C$. atlantica é suscetível ao fungo entomopatogênico $V$. lecanii.

A concentração de $1,0 \times 10^{8}$ conídios $/ \mathrm{mL}$ foi a mais eficiente e provocou efeito letal mais rapidamente que as demais concentração testadas.

A concentração de $1,0 \times 10^{8}$ conídios $/ \mathrm{mL}$ de V. lecanii causou mortalidade de $86 \%$ às ninfas de C. atlântica.

\section{REFERÊNCIAS BIBLIOGRÁFICAS}

ALVES, S. B. et al. Técnicas de laboratório. In: ALVES, S. Controle microbiano de insetos. 2 ed. Piracicaba: FEALQ, 1998. p.637711.

DREES, B.M. Aphid management [on line]. Texas: Texas Agricultural Extension Service, 1993. [10 april 2003]. Available from: World Wide Web: <URL: http://entowww.tamu.edu/extension/ bulletins/uc/uc-031.html.

FOURNIER, V.; BRODEUR, J. Biological control of lettuce aphids with the entomopathogenic fungus Verticillim lecamii in greenhouses. Integrated control in glasshouses [S.1]: IOBC/WPRS, 1999. p.77-80. (IOBC/WPRS Bulletin, v.22, n.1, 1999).

HAJEK, A.E.; ST. LEGER, R.J. Interactions between fungal pathogens and inset hosts. Annual Review Entomology, v.39, p.293322, 1994.

HALL, R.A. Control of whitefly, Trialeurodes vaporariorum and cotton aphid, Aphis gossypii in glasshouses by two isolates of the fungus, Verticillium lecanii. Annals of Applied Biology, v.101, n.1, p.1-11, 1982.

HALL, R.A.; BURGES, H.D. Control of aphids in glsshouses with the fungus, Verticillium lecanii. Annals of Applied Biology, v.93, n.2, p.235-246, 1979.

IEDE, E.T. et al. Ocorrência de Cinara pinivora (Homoptera: Aphidadae, Lachnidae) em reflorestamento de Pinus spp. no sul do Brasil. In: CONGRESSO BRASILEIRO DE ZOOLOGIA, 30., Recife, 1998. Anais... Recife: 1998. p.141.
KHACHATOURIANS, G.G. Production and use of biological pest control agents. TIBTECH, v.36, p.120-124, 1986.

LOUREIRO, E.S. Compatibilidade de fungos entomopatogênicos com outros produtos fitossanitários e sua interação com Myzus persicae (Sulzer, 1776), Aphis gossypii Glover, 1877 (Hemiptera, Aphididae) e Orius insidiosus (Say, 1832) (Hemiptera, Anthocoridae). 2001, 121f. Dissertação (Mestrado em Agronomia/Entomologia) Universidade Federal de Lavras, Lavras, 2001.

MILNER, R.J.; LUTTON, G.G. Dependence of Verticillium lecanii (Fungi: Hyphomycetes) on higt humidities for infection and sporulation using Myzus persicae (Homoptera: Aphididae) as host.

Environmental Entomology, v.15, n.2, p.380-382, 1986.

MILNER, R.J. Prospects for biopesticides for aphid control. Entomophaga, Paris, v.42, n.2, p.227-239, 1997.

NEVES, P.M.J. Seleção de isolados de Beauveria bassiana e Metarhizium anisopliae e controle de Cornitermes cumulans (Kollar, 1832) (Isoptera, Termitidae). 1998. $113 \mathrm{f}$. Tese (Doutorado em Ciências Biológicas/ Entomologia) - Escola Superior de Agricultura "Luiz de Queiroz", Piracicaba, 1998.

OLIVEIRA, N.C.; LOUREIRO, E.S.; WILCKEN, C.F. Ocorrência de Verticillium lecanii em populações do pulgão-do-Pinus Cinara atlantica em Pinus taeda no estado de São Paulo. In: CONGRESSO BRASILEIRO DE ENTOMOLOGIA, 2002, Manaus. AM. Anais... Manus: 2002, p.74.

OTTATI, E.L. Relatório de estágio curricular supervisionado realizado na Klabin - Fabricadora de Papel e Celulose S.A. Botucatu: Universidade Estadual Paulista "Julio de Mesquita Filho", 1999. 52p.

R. Árvore, Viçosa-MG, v.28, n.5, p.765-770, 2004 
PENTEADO, S.R.C. et al. Pulgão do Pinus - nova praga florestal. Série Técnica Ipef, v.13, n.33, p.97-102, 2000.

PENTEADO, S.R.C. et al. Ocorrência de Verticillium lecanii em populações de Cinara pinivora e Cinara atlantica, no Brasil. In: SIMPÓSIO DE CONTROLE BIOLÓGICO, 7., 2001, Lavras. Anais... Lavras: 2001. p.324.

QUINLAN, R.J. Use of fungi to control insects in glasshouses. In: BURGE, M. N. (Ed.), Fungi in biological control systems. Manchester: Manchester University Press, 1988. p. 19.

ROVESTI, L. et al. Use of entomopathogenic fungi for pest control in protected crops in Italy. Bulletin Section Regionale Ouest Paelartique, v. 20, n. 4, p. 285-292, 1997.
SOCIEDADE BRASILEIRADE SILVICULTURASBS. Estatísticas: área total reflorestada no Brasil 1998. [on line]. Piracicaba, s.d. [18 ago 2001]. Disponível de: Word Wide Web: <URL: http:// www.ipef.br/sbs/estatísticas.

STEENBERG, T.; HUMBER, R.A.

Entomopathogenic potential of Verticillium and Acremonium species (Deuteromycotina: Hyphomycetes). Journal of Invertebrate Pathology, v. 73, p. 309-314, 1999.

Van der SCHAAF, D.A.; MALAIS, M.;

RAVENSBERG, W.J. The use of Verticillium lecanii against whitefly and thrips in glasshouse vegetables in the Nethelands In:

INTERNATIONAL COLLOQUIUM ON INVERTABRATE PATHOLOGY AND MICROBIAL COTROL, 5., 1990, Adelaide. Proceedings...

Adelaide: Society of Invertebrate Pathology, 1990. p.391. 